\title{
MEDIASTINAL YOLK SAC TUMOR INFILTRATING THE HEART
}

\author{
R. Imaniar, E. Syahruddin, P.Z. Soepandi, A.C. Putra, F. Nurwidya* \\ Department of Pulmonology and Respiratory Medicine, Faculty of Medicine Universitas Indonesia, \\ Persahabatan Hospital, Jakarta 13230, Indonesia
}

\begin{abstract}
Background: As a rare tumor, yolk sac tumor is a type of neoplasm that appears like the yolk sac, extraembryonic mesenchyme, and allantois. The mediastinum is the second most frequent area after the gonadal area. Case report: We present an extremely rare case of 15 years old boy with mediastinal yolk sac tumor. The boy came with the chief complaint of swelling of the neck and face. Computed tomography scan of the chest revealed bulky mass with a cystic component that infiltrated the heart. The diagnosis of mediastinal yolk sac tumor was made after core needle biopsy. Histopathologic analysis revealed tumor mass with solid and microcystic structure and pleomorphic nucleus within the tumor cells. Laboratory finding showed elevated serum alpha-fetoprotein level reaching more than $8000 \mathrm{ng} / \mathrm{ml}$. Echocardiography revealed a mass in the right atrium. The patient condition was rapidly deteriorated due to his vena cava superior syndrome. Unfortunately, the patient died two days after diagnosis and we did not have the chance to do the therapy. Conclusion: Mediastinal yolk sac tumor is a rare malignancy that requires comprehensive management. The diagnosis should be made based on histopathological findings with the addition of thoracic computer tomography scan to assess the degree of infiltration to surrounding organ. A life-threatening condition such as vena cava superior syndrome should be assessed promptly to allow for immediate treatment.
\end{abstract}

Key Words: mediastinal yolk sac tumor, heart infiltration.

Germ cell tumors (GCTs) are rare tumors that arise from primordial germ cells [1]. The incidence of GCTs can be estimated at 0.9 per 100,000 children. In young men, malignant GCTs are the most common malignant tumor entity with an incidence of $7-8$ per 100,000 persons [2]. GCTs are usually located in gonadal sites (testis, ovarium). However, 1-5\% GCTs are of extragonadal origin with the most common primary site in the anterior mediastinum (50-70\%), followed by the retroperitoneal cavity, pineal gland, sacrococcygeal region, and vagina [2-5]. GCTs, regardless the site of the tumors, are classified into two categories: seminoma and nonseminomatous GCTs. Yolk sac tumor is nonseminomatous malignant GCTs that resembles yolk sac and extraembryonic mesenchyme [6]. Mediastinal yolk sac tumor is a malignant GCT which has a poor prognosis despite modern chemotherapy [5, 7]. In this report, we present a rare case of mediastinal yolk sac tumor in 15 years old boy that infiltrated the heart and formed a mass in the right atrium.

\section{CASE REPORT}

Fifteen years old boy came with his parents to our outpatient clinic with a chief complaint of swelling of his face and neck. The patient was admitted to regional hospital for one week due to progressive dyspnea and referred to our hospital for further diagnosis and treatment. The patient presented with a cough with whitish sputum, history of hemoptysis, loss of weights, and dysphagia. The physical examination revealed tachycardia, increased respiratory rate, isolated edema of the face and neck, elevated jugular venous pressure, the dull percussion of the

Submitted: April 23, 2016

*Correspondence: E-mail: fariz.nurwidya@gmail.com Abbreviations used: ECG - electrocardiogram; GCTs - germ cell tumors. left chest and decreased breath sound on the left lung.

The chest $\mathrm{x}$-ray showed homogeneous consolidation covering the left hemithorax that pushed the heart to the right. Computed tomography scan of the chest showed a bulky mass with a cystic component in the anterior mediastinum predominantly on the left hemithorax measuring $15 \times 12 \mathrm{~cm}$ in the axial plane and $15.2 \times 15 \mathrm{~cm}$ on the coronal plane (Fig. 1). This mass extended to the right hemithorax and compressed superior vena cava. The mass also infiltrated to the right atrium. The beta-human chorionic gonadotropin level was within the normal limit but the serum alphafetoprotein level was elevated to $>8000 \mathrm{ng} / \mathrm{ml}$. The electrocardiogram (ECG) revealed low QRS voltage suggestive of pericardial effusion. Echocardiography revealed a mass in the right atrium, pericardial effusion, with mild tricuspid regurgitation and mitral regurgitation (Fig. 2). Core needle biopsy was performed and diagnosis of mediastinal yolk sac tumor was made based on the histopathological finding. On the histopathologic examination, we found tumor mass with solid and microcystic structure. The tumor cells appeared to have polymorphic nuclei. We didn't find Schiller - Duval body which is pathognomonic for yolk sac tumors (Fig. 3).

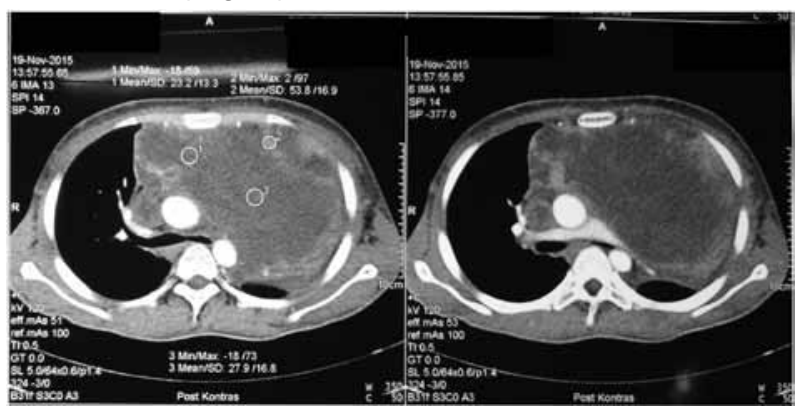

Fig. 1. Chest computed tomography showing a mass in the anterior of the mediastinum and infiltrate the right atrium 


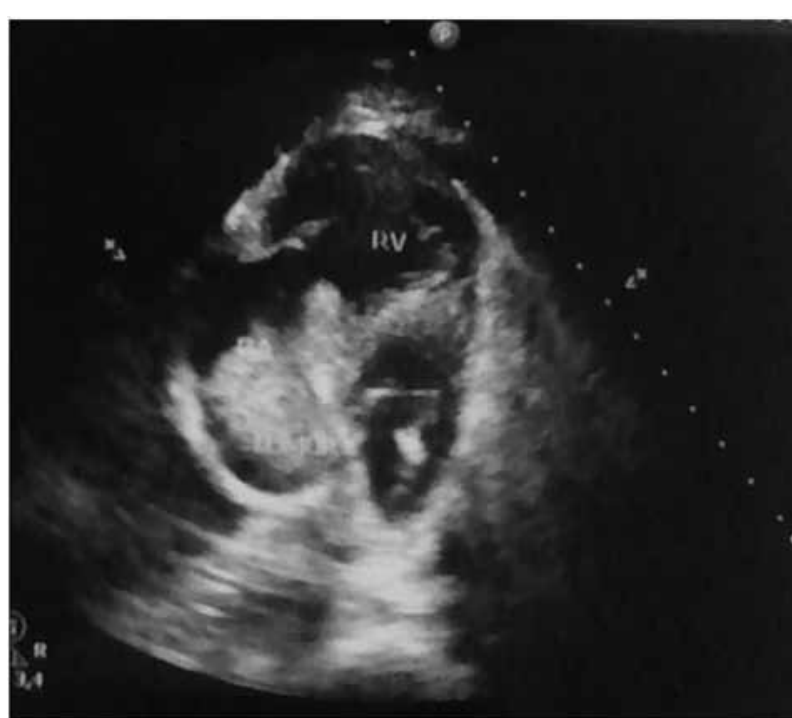

Fig. 2. Echocardiography revealed a mass in the right atrium

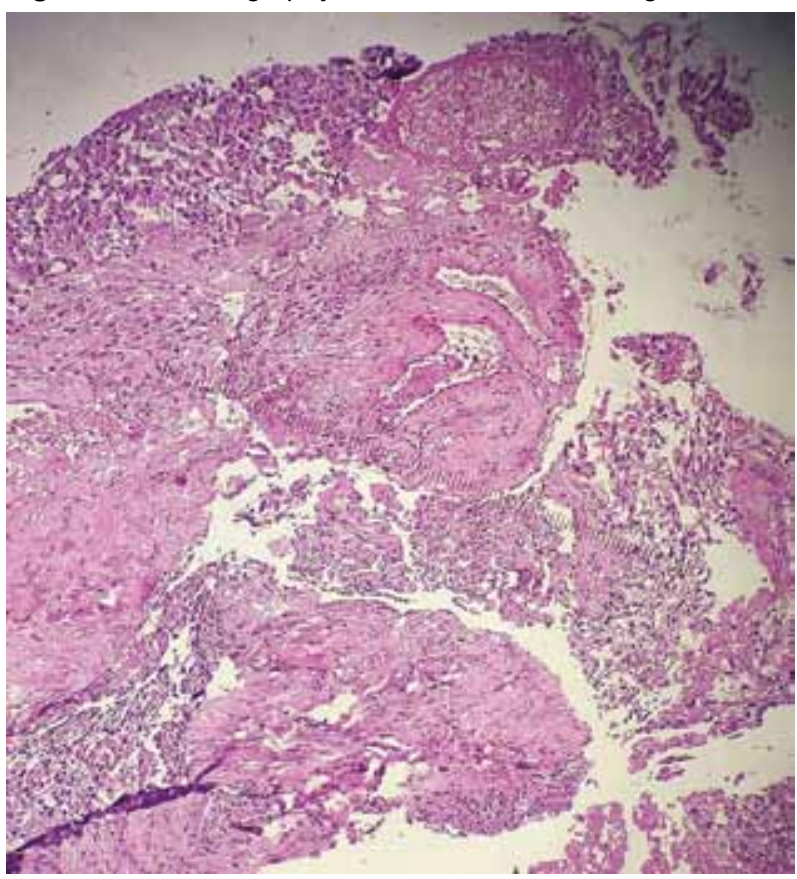

Fig. 3. Histopathologic examination showed tumor mass with solid and microcystic structure. The tumor cells have a pleomorphic nucleus, $\times 200$

Due to the superior vena cava syndrome, we performed elevation of the head and neck, administered supplemental oxygen, intravenous corticosteroid, and performed five fraction of radiotherapy (total of $1500 \mathrm{cGy}$ ) to the tumor location. The patient responded well to the treatment. The jugular venous pressure decreased, the swelling of the face and neck improved, and shortness of breath alleviated. The radiotherapy was discontinued despite clinical improvement because the evaluation of the chest $x$-ray revealed there were no objective signs of tumor shrinkage. We planned to do 3-4 cycles of chemotherapy with a platinum-based regimen to the patient and evaluate the serum alpha-fetoprotein serum level for eligibility for surgery. We did not have the chance to do the chemotherapy because the patient died two days after diagnosis was made. The patient's family did not give us permission to do the autopsy.

\section{DISCUSSION}

We presented a case of yolk sac tumor in the anterior mediastinum of a 15 years old boy. Yolk sac tumor is a malignant GCT that occurs predominantly in male age 10 to 40 years and mostly found in gonadal sites [8]. However, rare cases of mediastinal yolk sac tumor in 2 years old girl and 66 years old women were also reported $[8,9]$. Yolk sac tumor can also be found in extragonadal sites, most frequently in the anterior mediastinum. The patient was referred to Persahabatan Central General Hospital from a regional hospital with superior vena cava syndrome. Previously, the patient presented with progressive dyspnea over 2 weeks before superior vena cava syndrome developed. Patients with mediastinal GCTs usually present with dyspnea (25\%), chest pain (23\%), cough (17\%), followed by fever ( $13 \%)$, weight loss (11\%), vena cava superior syndrome (6\%), fatigue/weakness (6\%), and pain at other location except the chest $(5 \%)$. Less frequent symptoms were chest wall or cervical mass $(2 \%)$, and hemoptysis, hoarseness, nausea, or dysphagia ( $1 \%$ each) [3].

The computed tomography scan revealed the bulky mass with cystic component infiltrating to the right atrium. Echocardiography confirmed the findings and revealed pericardial effusion. These findings suggest cardiac involvement. Yolk sac tumor is very aggressive, includes locally advanced and metastatic tumors at diagnosis [10,11]. Several articles reported cases of yolk sac tumor (gonadal and extragonadal sites) with cardiac metastases [12-15]. To our knowledge, direct spread of mediastinal GCTs to the heart has never been reported before so this case is the first case such involvement has ever been reported.

The tumor can spread to the heart by four mechanisms: (1) direct extension, (2) through the bloodstream, (3) through the lymphatic system, (4) intracavitary diffusion through either superior vena cava or pulmonary veins. The clinical findings of cardiac involvement in cancer are highly variable. Most frequently, cardiac involvement goes unrecognized and only diagnosed until after death. The findings of cardiac involvement in our patient were found accidentally by computed tomography scan and echocardiography [15].

Symptoms caused by cardiac involvement in cancer depend on the location of the tumor. Focal lesion secondary to the myocardium probably does not produce any symptoms. Pericardial involvement can produce increasing shortness of breath, hypotension, tachycardia, and clinical findings of cardiac tamponade. Pericardial effusion is often the first clinical manifestation of pericardial involvement. In our patient, pericardial effusion is evident in ECG which showed low QRS voltage and appeared on echocardiography. If the tumor infiltrates the wall of the heart it may produce arrhythmias, conduction disturbances, and complete atrioventricular blocks. Ventricular involvement may cause heart failure due to systolic or diastolic dysfunction. Left atrial involvement may cause thrombi that 
facilitate distant metastasis and cause an ischemic lesion in the systemic arterial system. Right atrial involvement may cause thrombi that cause pulmonary emboli to the lung [15].

Our patient had increased serum alpha-fetoprotein and normal human chorionic gonadotropin level. These findings correspond with the nature of yolk sac tumor. Alpha-fetoprotein has been found in the serum, in the cytoplasm of the tumor cells, and in the eosinophilic globules by the immunohistochemical techniques in yolk sac tumors [10]. Alpha-fetoprotein is also used as a marker for evaluation of treatment and as a prognostic marker for the tumor [2-16].

Our patient came with superior vena cava syndrome so we performed radiotherapy to the tumor location to release compression of the tumor to the superior vena cava. The radiotherapy was discontinued despite clinical improvement because there was no evident of objective shrinkage of the tumor. The mediastinal yolk sac tumor diagnosis was made after we performed core needle biopsy. Unfortunately, the patient died two days after diagnosis and we did not have the chance to administered chemotherapy. Current therapy of poor prognosis non-seminoma GCTs based on European Germ Cell Cancer Consensus Group (EGCCCG) are 4 cycles of PEB (cisplatin, etoposide, and bleomycin) chemotherapy followed by evaluation of the tumor markers. Resection of the residual tumor can be done if the marker is normalized and the tumor is resectable. If the tumor marker is elevated but reached a plateau level, follow-up is indicated in 4-12 weeks. If there is no increase in tumor marker level, resection can be performed. However, if there is an increase in tumor marker level, salvage chemotherapy is indicated [16]. Primary mediastinal yolk sac tumor is classified as poor prognosis by the International Germ Cell Cancer Collaborative Group (IGCCCG) with the 5-year survival of 50\% [7].

In another case report, it is showed that pre-operative chemotherapy of PEB, followed by complete excision of all residual anterior mediastinal mass resulted in complete response for 5 years [5]. In our case, however, the patient presented with swelling of his face and neck and severe respiratory distress in the last one week that represent superior vena cava syndrome. Moreover, the tumor has already infiltrated the heart in our patient. This deteriorating condition did not allow us to start the chemotherapy, instead, radiotherapy was considered the appropriate options to relieve the symptoms at that moment.

In conclusion, primary mediastinal yolk sac tumor is an aggressive tumor that often invades and infiltrates surrounding structures and metastasis. We presented a unique case in which the tumor invaded the heart and formed mass inside the right atrium. The patient initially responded to the radiotherapy but then his condition declined rapidly due to his severe vena cave superior syndrome. In our case, we did not have the chance to do the chemotherapy treatment. Early diagnosis and treatment are important in the management of malignant GCTs. However, the prognosis of primary mediastinal yolk sac tumor is poor and further research is needed to find the best treatment modality and regimen for the tumor.

\section{REFERENCES}

1. Chaganti RSK, Rodriguez E, Mathew S. Origin of adult male mediastinal germ cell tumors. Lancet 1994; 343: 1130-2.

2. Göbel U, Schneider DT, Calaminus G, et al. Germcell tumors in childhood and adolescence. Ann Oncol 2000; 263: $263-71$.

3. Bokemeyer C, Nichols CR, Droz JP, et al. Extragonadal germ cell tumors of the mediastinum and retroperitoneum: results from an international analysis. J Clin Oncol 2002; 20: 1864-73.

4. Albany C, Eihnorn LH. Extragonadal germ cell tumors: clinical presentation and management. Curr Opin Oncol 2013; 25: 261-5.

5. Akasbi Y, Najib R, Arifi S, et al. Complete histologic response to chemotherapy in a patient with a mediastinal yolk sac tumor: a case report. BMC Research Notes 2014; 7: 803 .

6. Ueno T, Tanaka YO, Nagata M, et al. Spectrum of germ cell tumors: from head to toe. Radiographics 2004; 24: $387-404$.

7. International Germ Cell Cancer Collaborative Group (IGCCCG). The International Germ Cell Consensus Classification: a prognostic factor based staging system for metastatic germ cell cancer. J Clin Oncol 1997; 15: 594-603.

8. Uchiyama M, Iwafuchi M, Matsuda Y. Mediastinal yolk sac tumor in a young girl: case report and review of literature. J Pediatr Surg 1996; 31: 1316-21.

9. Coscun U, Günel N, Yildrim Y, et al. Primary mediastinal yolk sac tumor in a 66-year-old woman. Med Principles Pract 2002; 11: 218-20.

10. Dehner LP. Gonadal and extragonadal germ cell neoplasia of childhood. Hum Pathol 1983; 14: 493-511.

11. Akutsu N, Adachi Y, Isosaka M, et al. Mediastinal yolk sac tumor producing protein induced by vitamin $\mathrm{K}$ absence or antagonist-II. Intern Med 2015; 54: 1531-6.

12. Nunes MCP, Moreira DR, Ferrari TCA. Cardiac metastasis from yolk sac tumor: a case report and review. Exp Hematol Oncol 2013; 2: 13.

13. Vohra A, Saiz E, Davila E, et al. Metastatic germ cell tumor to the heart presenting with syncope. Clin Cardiol 1999; 22: 429-33.

14. Avasthi R, Chaudary ST, Mohanty D, et al. Testicular mixed germ cell tumor metastasizing to the heart. JAPI 2008; 56: $812-5$.

15. Bussani R, De-Giorgio F, Abbate A, et al. Cardiac metastases. J Clin Pathol 2007; 60: 27-34.

16. Schmoll HJ, Souchon R, Krege S, et al. European consensus on diagnosis and treatment of germ cell cancer: a report of the European Germ cell Cancer Consensus Group (EGCCCG). Ann Oncol 2004; 15: 1377-99. 